Pacific Journal of Mathematics

THE DIRICHLET PROBLEM FOR NONLINEAR ELLIPTIC 


\title{
THE DIRICHLET PROBLEM FOR NONLINEAR ELLIPTIC EQUATIONS
}

\author{
Jane Cronin
}

1. Introduction. It is often convenient to divide the question of solving the Dirichlet problem for a nonlinear elliptic differential equation into two parts:

(A) The "local" problem, that is, the problem of solving the equation for boundary functions ${ }^{1}$ sufficiently "close" to the boundary function of a given solution.

(B) The "extension" problem, that is, the problem of finding solutions corresponding to a given set of boundary functions if the solution for one boundary function in the set is known.

S. Bernstein takes this approach in his fundamental papers on nonlinear elliptic equations $[1,2]$, and in other papers (See [7] for a bibliography.) this viewpoint is used more or less explicitly.

Problem (B) is essentially the profound and difficult problem of finding "a priori" estimates for solutions of nonlinear elliptic equations (cf.the papers by Bernstein $[1,2]$, Schauder [15], Leray $[10,11]$, and Nirenberg [13]. Nirenberg gives, besides important new results, a clear account of previous work in the subject.) We shall be concerned here only with problem (A), which is much simpler.

In solving (A), the usual procedure is to invoke an assumption which implies uniqueness of solution, that is, an assumption which implies that the corresponding Jacobi equation has only the zero solution. This assumption is used to prove that there is a solution for each boundary function which is sufficiently "close" to the boundary function of the given solution. If the uniqueness hypothesis is relinquished, it may turn out that the equation has several solutions or no solutions at all for some boundary function which neighbors the boundary function of the given solution $[4,12]$. But this is a statement about real solutions of the differential equation. We shall show here that if complex

\footnotetext{
${ }^{1}$ We shall refer to a set of boundary values as a boundary function.

Received October 11, 1953.
}

Pacific J. Math. 5 (1955), 335- 344 
solutions are admitted, then the differential equation always has at least one solution for each boundary function sufficiently close to the boundary function of the given solution. We obtain a lower bound for the number of distinct solutions, and we obtain a sufficient condition that there exist a real solution of the differential equation.

More precisely, we consider the equation

$$
F(x, y, z, p, q, r, s, t)=\psi(x, y),
$$

where $F$ is analytic in all its variables. (For simplicity of notation, we consider the case of two independent variables. All the results hold for the case of $n$ variables.) Let $R$ be a bounded connected region in the plane with a nice boundary $S .^{2}$ The normed rings $E_{\alpha, n}$ and $e_{a, n}$, which consist of functions on the domains $R \cup S$ and $S$, respectively, having $\alpha$-Hölder continuous $n$th derivatives, are defined in the usual way. Suppose $(E)$ has a solution $z_{0} \in E_{a, 3}$ with boundary function $\phi_{0}(\xi) \in e_{a, 3}$ when $\psi=\psi_{0} \in E_{a, 1}$. Assume that $F$ is elliptic relative to $z_{0}$ and that $z_{0}$ is an isolated solution of (E) when $\psi=\psi_{0}$. Our purpose is to solve the following Dirichlet problem:

Given $\psi(x, y) \in E_{a, 1}$ and boundary function $\phi(\xi) \in e_{a, 3}$, such that $\psi-\psi_{0}$ and $\phi-\phi_{0}$ are both sufficiently small in terms of the norms of $E_{a, 1}$ and $e_{\alpha, 3}$, respectively, does $(\mathrm{E})$ have a solution $z(x, y) \in E_{a, 3}$ with $\left.z\right|_{S}=\phi$ ?

For completeness, we state first a well-known theorem.

THE OREM. If the Jacobi equation associated with (E), that is,

$$
\left[F_{r}\right]_{0} u_{x x}+\left[F_{s}\right]_{0} u_{x y}+\left[F_{t}\right]_{0} u_{y y}+\left[F_{p}\right]_{0} u_{x}+\left[F_{q}\right]_{0} u_{y}+\left[F_{z}\right]_{0} u=0
$$

(where $\left[F_{r}\right]_{0}$ means $F_{r}$ evaluated at $z=z_{0}$ ), has only the zero solution with boundary function $\phi \equiv 0$, then ( $\mathrm{E})$ has a unique solution $z(x, y) \in E_{a, 3}$ for all $\psi, \phi$ sufficiently close (in the appropriate norm) to $\psi_{0}$ and $\phi_{0}$.

Now suppose $(\mathrm{J})$ has $n$ linearly independent solutions with boundary function $\phi \equiv 0$. Then we have:

THE OREM B. Equation (E) has at least one solution of the form

$$
u(x, y)+i v(x, y)
$$

\footnotetext{
${ }^{2}$ For a precise formulation of the conditions on $S$, see $[5$, footnote (4)].
} 
where $u(x, y), v(x, y) \in E_{a, 3}$ for all $\psi, \phi$ sufficiently close to $\psi_{0}, \phi_{0}$. For a good many pairs ${ }^{3} \psi, \phi$, equation (E) has at least $2^{n}$ distinct solutions of this form.

Theorems $\mathrm{A}$ and $\mathrm{B}$ are direct extensions of the theorem on the continuity of roots $[9, \mathrm{p} .122]$ of algebraic equations.

In order to investigate whether $(\mathrm{E})$ has any real solutions, we use a concept of multiplicity that will be described in a later section of this paper.

THEOREM C. If the multiplicity of the solution $z_{0}$ is odd, equation ( $\mathrm{E}$ ) has at least one real solution, that is, a solution in $E_{a, 3}$, for all $\psi, \phi$ sufficient* ly close to $\psi_{0}, \phi_{0}$.

Theorem $\mathrm{C}$ is a direct extension of the elementary theorem that a polynomial of odd degree with real coefficients has at least one real solution.

Instead of proving Theorems A, B, C directly, we shall study a functional equation of which $(\mathrm{E})$ is a special case. Theorems $\mathrm{A}, \mathrm{B}$, and $\mathrm{C}$ are special cases of existence theorems which are obtained for the functional equation.

2. The existence theorems. Let $\mathfrak{X}$ be a Banach space over the real numbers. We consider the equation

$$
(I+C+T) x=y,
$$

where $x, y \in \mathfrak{X}, l$ is the identity, $C$ is linear completely continuous, and $T$ satisfies the hypotheses:

(1) $T(0)=0$.

(2) There is a neighborhood $U$ of 0 and a positive constant $B$ such that $u, v \in U$ implies

$$
\|T(u)-T(v)\|<B[\|u\|+\|v\|][\|u-v\|] .
$$

(3) $T$ is an analytic function of $x[8, p .81]$.

It is clear that $x=0$ is a solution of (1) when $y=0$. We assume that this is an isolated solution. The problem is to investigate the solutions $x$ of (1) for given small $y$. To avoid repetition, we use the notation of [3].

In $[4]$ and $[5]$, it was shown that studying the solutions of $(E)$ is cquivalent

${ }^{3}$ See [4, Definition 3.1] for a definition of the term "good many." 
to studying the solutions of a special case of equation (1). The proof given in [4] is shorter and clearer.

If $(I+C)$ is nonsingular, equation $(1)$ has a unique local solution as shown in [3]. (Theorem $\mathrm{A}$ is a special case of this statement.) If $(l+C)$ is singular, we consider possible complex solutions by studying a complex extension of (1). First we define a complex extension $\mathbb{Y}$ of the real Banach space $\mathfrak{X}$. This $\mathfrak{O}$ is a complete metric space which is also a linear space over the complex numbers. The elements of $\mathbb{D}$ are pairs $(u, v)$ of elements of $X$. Addition in $\mathcal{V}$ is defined by

$$
(u, v)+(x, y)=(u+x, v+y),
$$

and multiplication by complex numbers $(\alpha, \beta)$ is defined by

$$
(\alpha, \beta)(u, v)=(\alpha u-\beta v, \beta u+\alpha v) .
$$

REMARK. Any element $(u, v) \in \bigvee$ may be written as the linear combination

$$
(u, v)=(1,0)(u, 0)+(0,1)(v, 0) .
$$

The metric $\rho$ of $\mathfrak{Y}$ is defined in terms of the norm in $\mathfrak{X}$ by

$$
\rho\left[(u, v),\left(u_{0}, v_{0}\right)\right]=\left\|u-u_{0}\right\|+\left\|v-v_{0}\right\| .
$$

Since $\mathfrak{X}$ is complete, $\mathscr{Y}$ is complete. Also $\mathfrak{C}^{\mathfrak{l}}$ is an extension of $\mathfrak{X}$, that is, $\mathfrak{X}$ is topologically isomorphic to the subset $\mathfrak{T}$ of $\mathfrak{C}$ which consists of elements of the form $(u, 0)$. By a real element of $\mathfrak{N}$, we shall mean an element of $\mathfrak{T} \equiv \mathfrak{X}$.

Using the Remark, we define a completely continuous operator $C$ on $\overbrace{c}^{n}$ by

$$
C[(u, v)]=C[(1,0)(u, 0)+(0,1)(v, 0)]=(1,0)(C u, 0)+(0,1)(C v, 0) .
$$

Similarly, the linear operators $\varepsilon_{1}, \mathcal{E}^{1}$, and $R$ are defined by:

$$
\begin{aligned}
& \varepsilon_{1}[(u, v)]=(1,0)\left(E_{1} u, 0\right)+(0,1)\left(E_{1} v, 0\right), \\
& \mathcal{E}^{1}[(u, v)]=(1,0)\left(E^{1} u, 0\right)+(0,1)\left(E^{1} v, 0\right),
\end{aligned}
$$

and

$$
R[(u, v)]=(1,0)(R u, 0)+(0,1)(R v, 0) .
$$

From these definitions, it is clear that $C, \varepsilon_{1}, \varepsilon^{1}$, and $R$ are extensions in $\mathcal{C}^{\mathfrak{C}}$ of $C, E_{1}, E^{1}$, and $R$ respectively; that is, 


$$
C / X=C, \varepsilon_{1} / X=E_{1}, \varepsilon^{1} / X=E^{1} \text {, and } R / X=R \text {. }
$$

Moreover, it follows at once from the corresponding properties of $E_{1}, E^{1}$, and $R$ that $\varepsilon_{1}$ is a projection of $\stackrel{N}{S}$ on the null space of $I+C$, and that

$$
R(I+C)=I-\varepsilon_{1}=\varepsilon^{1} .
$$

Finally, we define an extension $\mathcal{J}$ in of transformation $T$. By $[8$, Theorem 4.5.1, p. 82 ] and Condition 2) on $T$, we have

$$
T(h)=\sum_{n=2}^{\infty} \frac{1}{n !} \delta^{n} T(0 ; h),
$$

where $\delta^{n} T(0 ; h)$ is a homogeneous polynomial of degree $n$ in $h$ [8, Theorem 4.3 .5$, p. 74]. Hence, by [8, Theorem 4.2.3, p. 66],

$$
\delta^{n} T(0 ; \alpha u+\beta v)=\sum_{\nu=0}^{n} \alpha^{\nu} \beta^{n-\nu} P_{\nu}[u, v]
$$

where each $P_{\nu}[u, v]$ is a homogeneous polynomial in $u, v$. Using the Remark, we define $\delta^{n} \mathrm{~g}[0 ;(u, v)]$ as

$$
\delta^{n} \Im[0 ;(u, v)]=\delta^{n} \mathfrak{J}[0 ;(1,0)(u, 0)+(0,1)(v, 0)]=\sum_{\nu=0}^{n}(0,1)^{n-\nu} P_{\nu}[u, v],
$$

and

$$
\exists(h)=\sum_{n=2}^{\infty} \delta^{n} \mathfrak{J}(0 ; h) .
$$

From the definition, it follows that:
(1) $r(0)=0$,
(2) $I$ is analytic,
(3) $\quad \exists / X=T$.

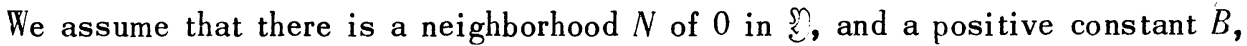
such that $(u, v),\left(u^{(1)}, v^{(1)}\right) \in N$ implies 


$$
\begin{array}{r}
\rho\left[\mathcal{J}(u, v)-\mathfrak{J}\left(u^{(1)}, v^{(1)}\right),(0,0)\right] \\
<B\left\{\rho[(u, v),(0,0)]+\rho\left[\left(u^{(1)}, v^{(1)}\right),(0,0)\right]\right\} \\
\left\{\rho\left[(u, v),\left(u^{(1)}, v^{(1)}\right)\right]\right\} .
\end{array}
$$

We also assume that $x=0$ is an isolated solution of the equation in $\underset{C}{\mathfrak{N}}$,

$$
(I+C+J) x=y,
$$

when $y=0$. Hence we can apply the theory of [3], [5], and [6] to study the solutions of (2). As was shown, the problem reduces to that of solving an equation in $\stackrel{c}{c}_{1}^{M}$, the null space of $l+C$.

$$
\varepsilon_{1} R g\left[x_{1}+F\left(x_{1}, y\right)\right]-\varepsilon R(y)=0 \text {. }
$$

In this case, equation ( 3 ) is a system of $n$ equations in $n$ complex unknowns,

$$
G_{i}\left(z_{1}, \cdots, z_{n}, y\right)=0 \quad(i=1, \cdots, n),
$$

where $G_{i}$ is analytic in the complex variables $z_{1}, \cdots, z_{n}$ and continuous in $y$, and $n$ is the dimension of $\mathfrak{C}_{1}$. We investigate the solutions of (4) by studying the topological index ${ }^{4}$ at zero of the mapping in complex Euclidean $r$-space

$$
G_{i}\left(z_{1}, \cdots, z_{n}, 0\right)=z_{i}^{\prime} \quad(i=1, \cdots, n) .
$$

In [6], it was shown that the index of this mapping is always $\geq 2^{n}$. (Actually a sharper lower bound was obtained, but we need not describe it here.) Hence, as in [3, Theorem 3.5], we obtain:

EXISTENCE THEOREM. For each sufficiently small y, equation (2) has at least one solution.

We call the index the multiplicity of the solution $x=0$ when $y=0$. It was shown in [4, Theorem 3.1] that the index or multiplicity tells the number of solutions in the complex case where the topological degree is always positive, that is, we have:

COUNTING THEOREM. If the index is $m$, then for a good many [4] sufficiently small $y$, equation (2) has exactly $n$ distinct solutions.

4 When referring to the topological index or to topological degree of a mapping in complex Euclidean $n$-space, we regard it as a mapping in real Euclidean $(2 n)$-space. 
Since $m \geq 2^{n}$, we have:

Cor Olla Ry. For a good many sufficiently small $y$, equation (2) has at least $2^{n}$ distinct solutions.

Theorem B is a special case of the Existence Theorem and this Corollary.

3. The real existence theorem. The two preceding theorems are about the solutions of (2), an equation in the complex linear space . These solutions of (2) can be regarded as the "complex" solutions of the original equation (1). However, we can also obtain information about solutions in $\mathfrak{X}$ of equation (1), that is, "real" solutions of (1). We prove a theorem that is analogous to the theorem that any polynomial equation of odd degree with real coefficients has at least one real solution.

REAL EXISTENCE THEOREM. If the index $m$ is odd, equation (1) has at least one solution $x \in \mathfrak{X}$ for each sufficiently small $y \in \mathfrak{X}$.

( Theorem $\mathrm{C}$ is a special case of this theorem.)

Proof. We show that if $y$ is real, then the complex extension of equation (1), that is, equation (2), has a "real" solution, that is, a solution in $X_{\text {. It }}$ is sufficient to show that if $y$ is real, equation (3) has a real solution $x_{1}$. For if $x_{1}$ is a real solution of $(3)$, then

$$
x_{1}+F\left(x_{1}, y\right)
$$

is a solution of $(2)$ that is in $\mathfrak{X}_{\text {. }}$ (The $F\left(x_{1}, y\right)$ is the solution, obtained by successive approximations, of

$$
x^{1}+\mathcal{E}^{1} R \zeta\left[x_{1}+x^{1}\right]-\varepsilon^{1} R(y)=0 .
$$

Since $y$ is real and

$$
\mathcal{E}^{1 / X}=E^{1}, R / \mathfrak{X}=R, \mathfrak{J} / \mathfrak{X}=T,
$$

then $F\left(x_{1}, y\right)$ is in $X_{\text {if }} x_{1}$ and $y$ are in $\left.X_{0}\right)$

As previously stated, the problem of solving (3) is that of solving a system of $n$ equations in $n$ unknowns

$$
G_{i}\left(z_{1}, \cdots, z_{n}, y\right)=0 \quad(i=1, \cdots, n),
$$


where each $G_{i}$ is analytic in $z_{1}, \cdots, z_{n}$, that is, each $G_{i}$ is a power series in $z_{1}, \cdots, z_{n}$. From the definitions of $\varepsilon_{1}, \varepsilon^{1}, R$, and $\mathcal{J}$, it follows that if $y$ is real then the coefficients in these power series are real. We complete the proof of the theorem by proving the following lemma.

INDEX LEMMA. Let

$$
M:\left(z_{1}, \cdots, z_{n}\right) \rightarrow\left(z_{1}, \cdots, z_{n}\right)
$$

be a mapping of complex Euclidean n-space into itself defined by the power series

$$
\sum a_{i}\left(k_{1}, \cdots, k_{n}\right) z_{1}^{k_{1}} \cdots z_{n}^{k_{n}}=z_{1}^{\prime} \quad(i=1, \cdots, n),
$$

where the $a_{i}\left(k_{1}, \cdots, k_{n}\right)$ are real numbers, and such that $M$ does not map $(0, \ldots, 0)$ into itself. Suppose the topological degree of $M$ at $(0, \ldots, 0)$ and relative ${ }^{5}$ to a sphere $S$ with center $(0, \cdots, 0)$ is $d$, an odd number. Then the system of equations

$$
\sum a_{i}\left(k_{1}, \cdots, k_{n}\right) z_{1}^{k_{1}} \cdots z_{n}^{k_{n}}=0 \quad(i=1, \cdots, n)
$$

has a real nonzero solution in $S$.

Proof of the Index Lemma. Since $d \neq 0$, system ( 5 ) has at least one solution in $S$. We assume that $(5)$ has no real solutions in $S$ and obtain a contradiction. Let

$$
E=[p \mid p \in \text { Interior of } S \text { and } M(p)=0]
$$

Suppose

$$
E_{j_{1}} \cdots j_{q}=\left[\left(\xi_{1}, \cdots, \xi_{n}\right)\right]
$$

is the set of points in $E$ such that the imaginary parts of the $\xi_{j_{1}}, \ldots, \xi_{j_{q}}$ are nonnegative and the imaginary parts of the other coordinates $\xi_{j}$ are negative. Since the coefficients $a_{i}\left(k_{1}, \cdots, k_{n}\right)$ are real, the set

$$
\bar{E}_{j_{1}} \cdots j_{q}=\left[\left(\bar{\xi}_{1}, \cdots, \bar{\xi}_{n}\right)\right]
$$

is also contained in $E$. Hence we may write

5 By the topological degree of a mapping $M$ relative to a set $S$, we mean the topological degree of $M / S$. 


$$
E=\bigcup_{i=1}^{l}\left(B_{i} \cup \overline{B_{i}}\right)
$$

where $B_{1}, \cdots, B_{n}$ are sets of the form $E_{j_{1}} \ldots j_{q}$, and $\bar{B}_{i}$ is the set of points conjugate to those in $B_{i}$, and $B_{1}, \ldots, B_{l}, \bar{B}_{1}, \ldots, \bar{B}_{l}$ are pairwise disjoint. Since (5) has no real solutions in $S$, there exist pairwise disjoint open sets $U_{1}, \ldots, U_{l}, \bar{U}_{1}, \ldots, \bar{U}_{l}$ such that $\bar{U}_{i}$ is the set conjugate to $U_{i}$, and for $i=$ $1, \cdots, n$,

$$
B_{i} \subset U_{i}, \bar{B}_{i} \subset \bar{U}_{i}
$$

According to a basic property of topological degree, we have

$$
d=\sum_{i=1}^{n}\left[d\left(U_{i}\right)+d\left(\bar{U}_{i}\right)\right],
$$

where $d\left(U_{i}\right)$ is the topological degree of $M$ at $(0, \ldots, 0)$ and relative to the open set $U_{i}{ }^{5}$

To complete the proof of the lemma, it is sufficient to show that $d\left(U_{i}\right)=$ $d\left(\bar{U}_{i}\right)$, for then (6) shows that $d$ is even, which contradicts the hypothesis. That $d\left(U_{i}\right)=d\left(\bar{U}_{i}\right)$ is a consequence of the definition of topological degree and the following remarks.

Suppose $\sigma$ is an $n$-simplex in complex Euclidean $n$-space; that is,

$$
\sigma=\left\langle p_{1}, \cdots, p_{n}\right\rangle,
$$

where $p_{j}=a_{j}+i b_{j}$ for $j=i, \cdots, n$, and

$$
\bar{\sigma}=\left\langle\bar{p}_{1}, \cdots, \bar{p}_{n}\right\rangle
$$

Regarded as $(2 n)$-simplexes in real Euclidean $(2 n)$-space, these simplexes are

$$
\sigma=\left\langle a_{1}, b_{1}, a_{2}, b_{2}, \cdots, a_{n}, b_{n}\right\rangle
$$

and

$$
\bar{\sigma}=\left\langle a_{1},-b_{1}, a_{2},-b_{2}, \cdots, a_{n},-b_{n}\right\rangle \text {. }
$$

Hence if $n$ is odd, $\sigma$ and $\bar{\sigma}$ have opposite orientations. Since the $a_{i}\left(k_{1}, \ldots, k_{n}\right)$ are real, we have $M(\bar{\sigma})=\overline{M(\sigma)}$. Hence $M(\sigma)$ and $M(\bar{\sigma})=\overline{M(\sigma)}$ have opposite 
orientations. If $n$ is even, $\sigma$ and $\bar{\sigma}$ have the same orientation. Also $M(\sigma)$ and $M(\bar{\sigma})=\overline{M(\sigma})$ have the same orientation.

\section{REFERENCES}

1. S. Bernstein, Sur la généralisation du problème de Dirichlet (Première partie) Math. Ann. 59 (1906), 253 - 271.

2. - Sur la généralisation du problème de Dirichlet (Deuxième partie) Math. Ann. 62 (1910), 82 - 136.

3. J. Cronin, Branch points of solutions of equations in Banach space, Trans. Amer. Math. Soc. 69 (1950), 208-231.

4. - Branch points of solutions of equations in Banach space II, Trans. Amer. Math. Soc. 76 (1954), 207-222.

5. The existence of multiple solutions of elliptic differential equations, Trans. Amer. Math. Soc. 68 (1950), $105-131$.

6. - Analytic functional mappings, Ann. of Math. 58 (1953), 175-181.

7. Enzyklopädie der Mathematischen Wissenschaften, II, 3.2.

8. E. Hille, Functional analysis and semi-groups, Amer. Math. Soc. Colloq. Publications, vol. XXXI, New York, 1948.

9. K. Knopp, Theory of functions, Part II, Dover Publications, New York, 1947.

10. J. Leray, Majoration des dérivées secondes des solutions d'un problème de Dirichlet, J. Math. Pures Appl. 17 (1938), 89-104.

11. — Discussion d'un problème de Dirichlet, J. Math. Pures Appl. 18 (1939), 249 - 284.

12. L. Lichtenstein, Untersuchungen über zweidimensionale reguläre Variationsprobleme I, Monatshefte für Mathematik und Physik 28 (1917), 3 - 51.

13. L. Nirenberg, On nonlinear elliptic partial differential equations and Hölder continuity, Comm. Pure Appl. Math. 6 (1953), 103 - 156.

14. J. Schauder, Über den Zusammenhang zwischen der Eindeutigkeit und Lös barkeit partieller Differentialgleichungen zweiter Ordnung vom elliptischen Typus, Math. Ann. 106 (1932), 661 - 721.

15. Über das Direchletsche Problem im Grossen für nicht-lineare elliptische Differentialgleichungen, Math. 37 (1933), 623-634.

16. E. Schmidt, Zur Theorie der linearen und nichtlinearen Integralgleichungen, Math. Ann. 65 (1907-1908), $370-390$.

\section{GEOPHYSICS RESEARCH DIRECTORATE AIR Force Cambridge Research Center}




\section{PACIFIC JOURNAL OF MATHEMATICS}

\section{EDITORS}

\author{
H.L. ROYDEN \\ Stanford University \\ Stanford, California \\ E. HEWITT \\ University of Washington \\ Seattle 5, Washington
}

\author{
R. P. DILWORTH \\ California Institute of Technology \\ Pasadena 4, California \\ * Alfred Horn \\ University of California \\ Los Angeles 24, California
}

\section{ASSOCIATE EDITORS}

\section{H, BUSEMANN \\ HERBERT FEDERER}

MARSHALL HALL

\section{P.R. HALMOS \\ HEINZ HOPF}

ALFRED HORN

\author{
R.D. JAMES \\ BØRGE JESSEN \\ PAUL LÉVY
}

GEORGE PÓLYA

J.J. STOKER

KOSAKU YOSIDA

\section{SPONSORS}

UNIVERSITY OF BRITISH COLUMBIA

UNIVERSITY OF SOUTHERN CALIFORNIA

CALIFORNIA INSTITUTE OF TECHNOLOGY

UNIVERSITY OF CALIFORNIA, BER KELEY

STANFORD RESEARCH INSTITUTE

STANFORD UNIVERSITY

UNIVERSITY OF CALIFORNIA, DAVIS

UNIVERSITY OF. UTAH

UNIVERSITY OF CALIFORNIA, LOS ANGELES

WASHINGTON STATE COLLEGE

UNIVERSITY OF CALIFORNIA, SANTA BARBARA

UNIVERSITY OF WASHINGTON

MONTANA STATE UNIVERSITY

UNIVERSITY OF NEVADA

OREGON STATE COLLEGE

AMERICAN MATHEMATICAL SOCIETY

HUGHES AIRCRAFT COMPANY

UNIVERSITY OF OREGON

SHELL DEVELOPMENT COMPANY

\section{UNIVERSTTY OF SOUTHERN CALIFORNIA}

Mathematical papers intended for publication in the Pacific Journal of Mathematics should be typewritten (double spaced), and the author should keep a complete copy. Manuscripts may be sent to any of the editors. Manuscripts intended for the outgoing editors should be sent to their successors. All other communications to the editors should be addressed to the managing editor, Alfred Horn, at the University of California Los Angeles 24, California.

50 reprints of each article are furnished free of charge; additional copies may be obtained at cost in multiples of 50 .

The Pacific Journal of Mathematics is published quarterly, in March, June, September, and December. The price per volume (4 numbers) is $\$ 12.00$; single issues, $\$ 3.50$; back numbers (Volumes $1,2,3$ ) are available at $\$ 2.50$ per copy. Special price to individual faculty members of supporting institutions and to individual members of the American Mathematical Society: $\$ 4.00$ per volume; single issues, $\$ 1.25$.

Subscriptions, orders for back numbers, and changes of address should be sent to the publishers, University of California Press, Berkeley 4, California.

Printed at Ann Arbor, Michigan. Entered as second class matter at the Post Office, Berkeley, California.

* During the absence of E.G. Straus.

UNIVERSITY OF CALIFORNIA PRESS - BERKELEY AND LOS ANGELES 


\section{Pacific Journal of Mathematics}

\section{Vol. 5, No. $3 \quad$ November, 1955}

Nesmith Cornett Ankeny and S. Chowla, On the divisibility of the class number of quadratic fields ............................. 321

Cecil Edmund Burgess, Collections and sequences of continua in the plane ........................................ 325

Jane Smiley Cronin Scanlon, The Dirichlet problem for nonlinear elliptic equations....................................... 335

Arieh Dvoretzky, A converse of Helly's theorem on convex sets ......... 345

Branko Grünbaum, On a theorem of L. A. Santaló................ 351

Moshe Shimrat, Simple proof of a theorem of P. Kirchberger .......... 361

Michael Oser Rabin, A note on Helly's theorem . ................... 363

Robert E. Edwards, On factor functions . . ................... 367

Robert E. Edwards, On certain algebras of measures ............... 379

Harley M. Flanders, Methods in affine connection theory.............. 391

Alfred Huber, The reflection principle for polyharmonic functions ........ 433

Geoffrey Stuart Stephen Ludford, Generalised Riemann invariants ....... 441

Ralph Gordon Selfridge, Generalized Walsh transforms............. 451 